\title{
Effect of Endurance Exercise Training and Gallic Acid on Tumor Necrosis Factor- $\alpha$ in an Animal Model of Alzheimer's Disease
}

\author{
Yunes Bazyar ${ }^{1}$, Samaneh Rafiei ${ }^{1}$, Ali Hosseini ${ }^{1}$, Mohammad Amin Edalatmanesh ${ }^{2 *}$ \\ ${ }^{1}$ Department of Exercise Physiology, Marvdasht Branch, Islamic Azad University, Marvdasht, Iran \\ ${ }^{2}$ Department of Physiology, College of Sciences, Shiraz Branch, Islamic Azad University, Shiraz, Iran
}

\section{ABSTRACT}

Introduction: Alzheimer's disease $(\mathrm{AD})$ is a non-refundable gradual neuro-degenerative disorder, in which the neurons, especially the ones in the memory zone, are damaged and cause increase in the level of cytokines, such as tumor necrosis factor- $\alpha$ (TNF- $\alpha$ ). In the present study, we investigated the effect of endurance exercise training and chronic administration of Gallic acid (GA) on the TNF- $\alpha$ level in rat hippocampus in the Trimethyltin (TMT)-treated model of AD. Materials and Methods: Seventy female Spraque Dawley rats were divided into seven groups: 1.Control, 2. AD, 3. GA50, 4. GA100, 5. Exercise, 6. Exercise+GA50, and 7. Exercise + GA100. In order to induce AD, Trimethyltin $(8 \mathrm{mg} / \mathrm{kg})$ was injected intraperitoneally to the rats in groups 2-7. Rats in the groups 5, 6, and 7 carried out an eight-week exercise program on a motorized treadmill (15-20 m/min, 0\% inclination for 15-30 $\mathrm{min} /$ day, and 5 days/ week). Animals of the groups 3 and 6 were treated by $50 \mathrm{mg} / \mathrm{kg}$ of GA and animals of groups 4 and 7 were treated by $100 \mathrm{mg} / \mathrm{kg}$ of the GA daily, for 2 weeks. Then, the TNF- $\alpha$ level in the hippocampus were measured. Results: The results indicated that the TNF- $\alpha$ level in the hippocampus was decreased in all test groups compared to the AD group. Conclusion: Our findings indicate that endurance exercise training, GA consumption, and both administration of GA and co-treatment with training have immunomodulatory effects and could be used to inhibit the cytokine release after TMT intoxication.

\section{Key words:}

1. Alzheimer Disease

2. Exercise

3. Gallic Acid

4. Hippocampus

* Corresponding Author: Mohammad Amin Edalatmanesh

E-mail: amin.edalatmanesh@gmail.com 


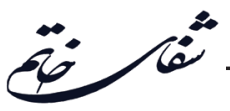

\section{اثر تمرين ورزشى استقامتى و اسيد كاليك بر فاكتور نكروز دهندة تومور آلفا در يك مدل حيوانى بيمارى آلزايمر}

يونس بازيار'، سمانه رفيعى'، على حسينى'، محمد امين عدالت منش"r"

'كروه فيزيولوزى ورزشى، واحد مرودشت، دانشكاه آزاد اسلامى، مرودشت، ايران

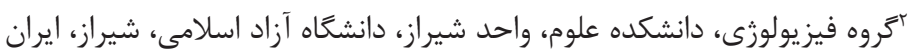

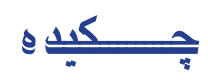

مقدمه: بيمارى آلزايمر يك اختلال تخريبكننده عصبى غيرقابل بازگشت تدريجى است كه در آن آن

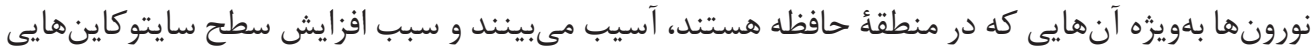

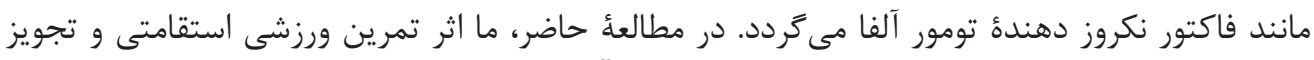

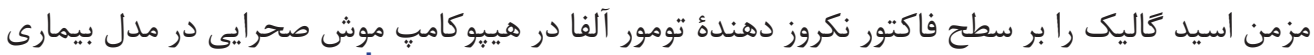

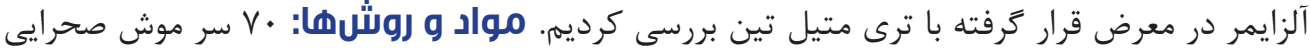

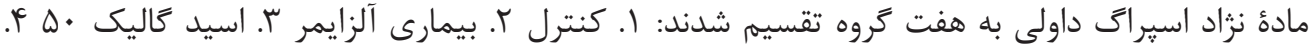

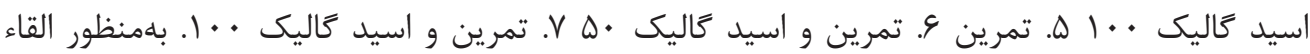

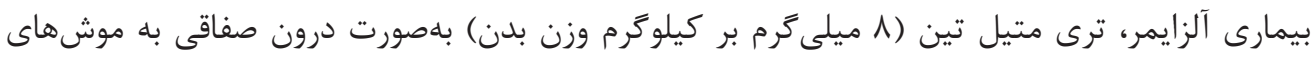

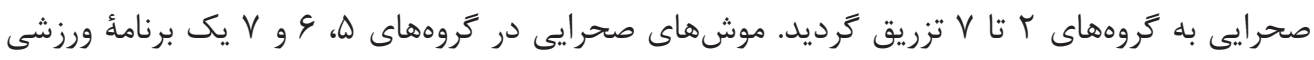

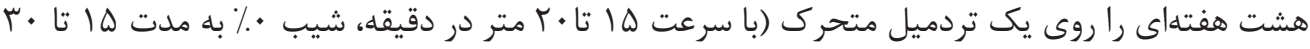

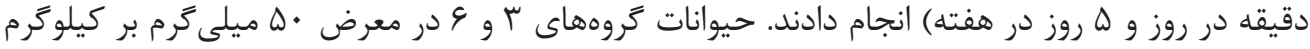

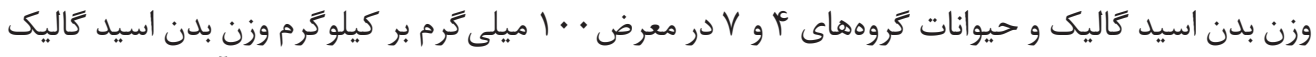

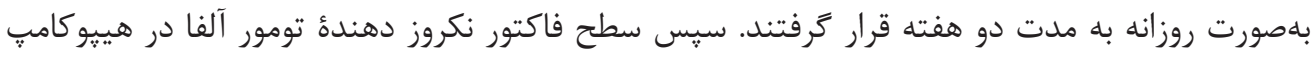

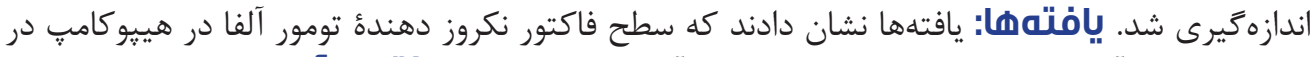

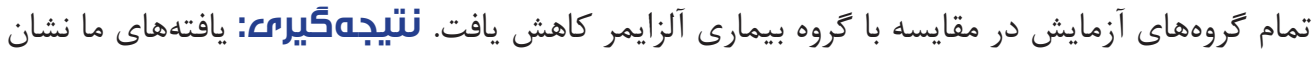

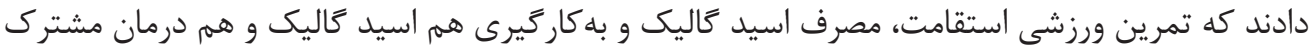

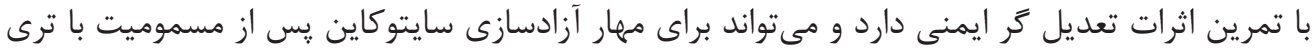
متيل تين استفاده تردد.

" نويسنده مسئول: محمد امين عدالت منش آنش آدرس الكترونيكى: amin.edalatmanesh@gmail.com 
سلولها مىشود ( • (). مهرترين عامل دفاعى عليه راديكال هاى (رئي

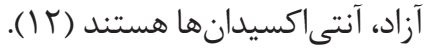

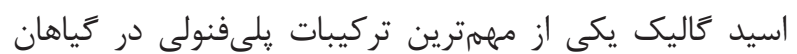

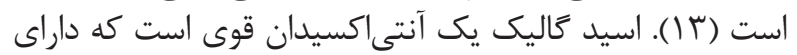

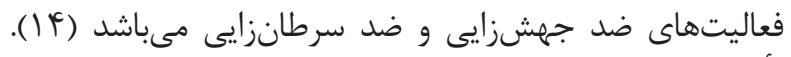
تأثيرات حفاظت عصبى بسياري

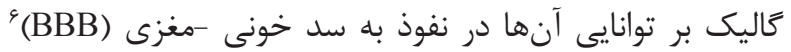

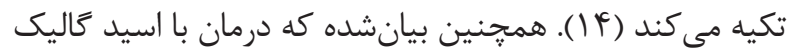

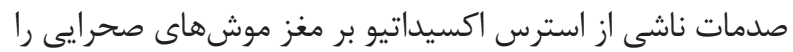

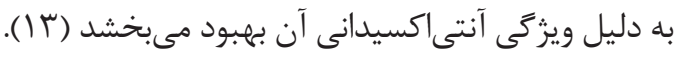

يروهشها نشان دادند كه فعاليتهاى ورزشى اثرات ضد التهن التهاب

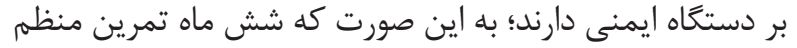
باعث كاهش سايتوكاين TNF-

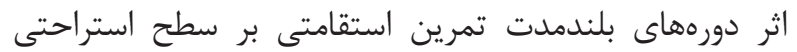

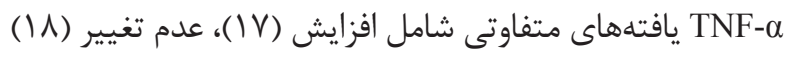

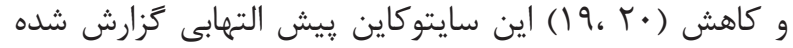

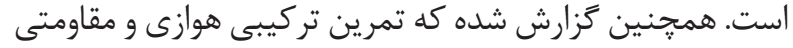

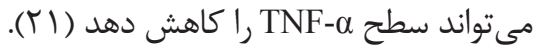

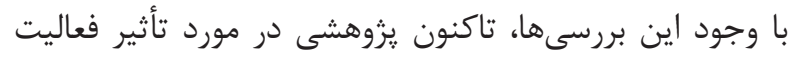

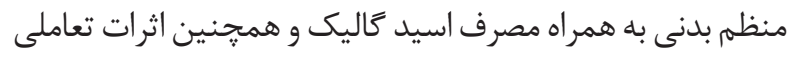

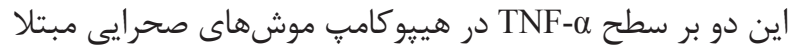

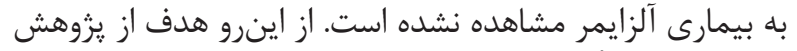

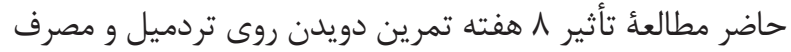

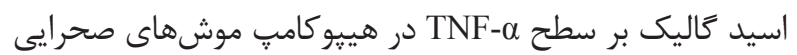
ماده مبتلا به بيمارى آلزايمر مى باشد.

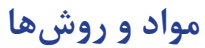
حيوانات

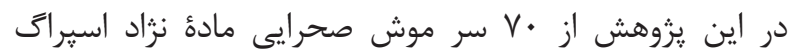

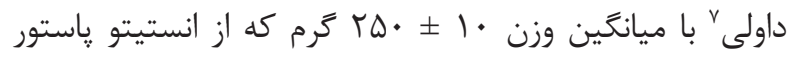

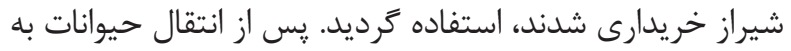

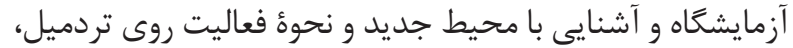

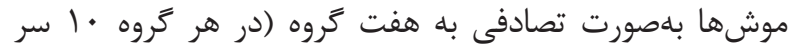

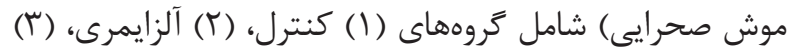

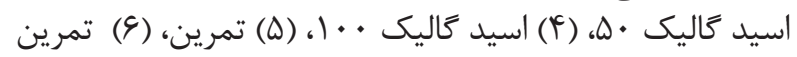

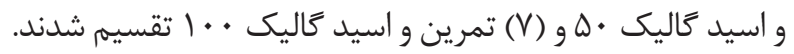

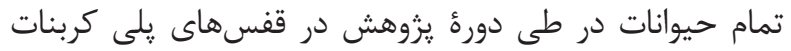

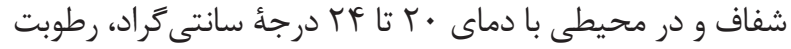

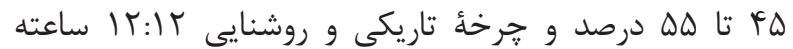

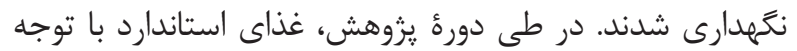

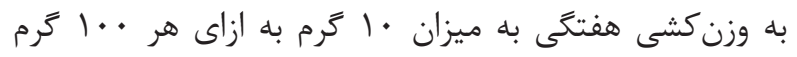

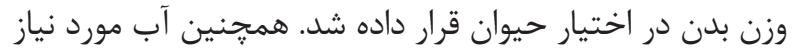

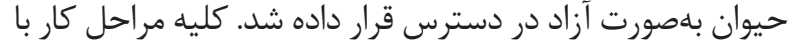

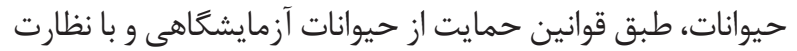

\section{${ }^{1}$ Microglia}

${ }^{2}$ Oxidative stress

${ }^{3}$ Tumor necrosis factor alpha

${ }^{4}$ Neurodegenerative disease

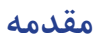

بيمارى آلزايمر يك اختلال مغزى ييشرونده است كه باطورو

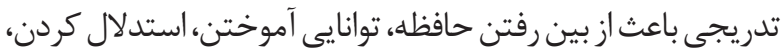

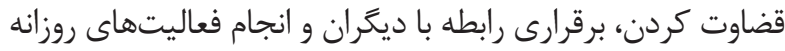

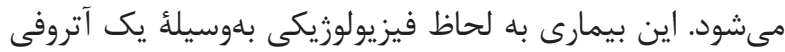

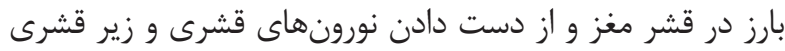

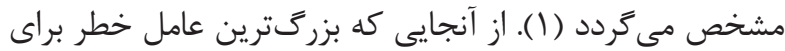

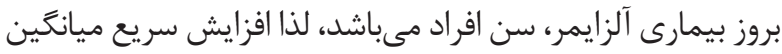

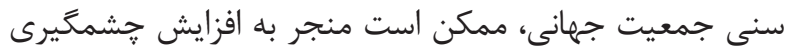

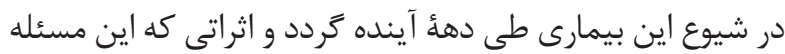

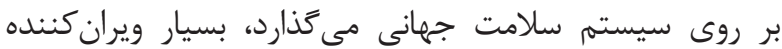

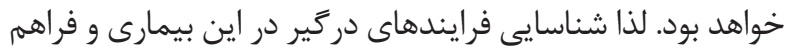

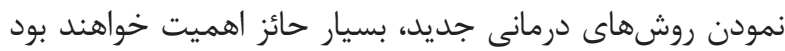

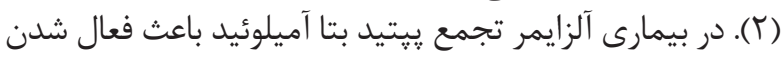

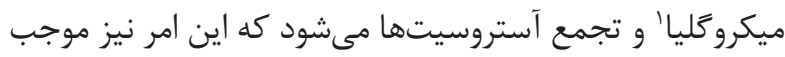

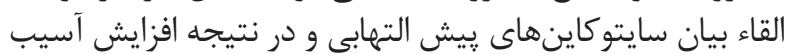

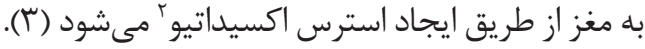

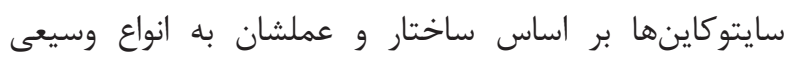

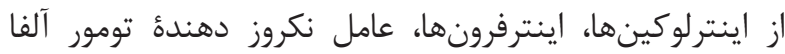

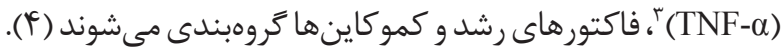

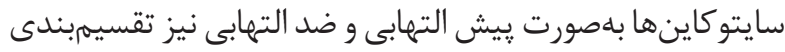
مى كردند.

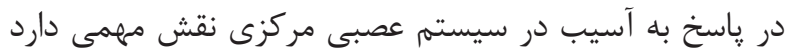

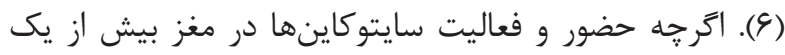

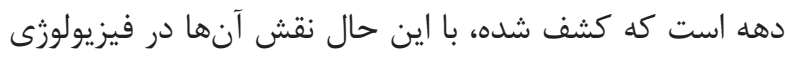

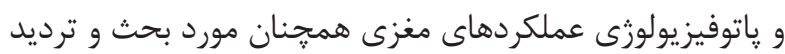

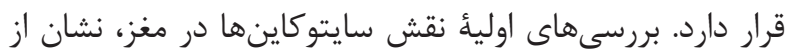

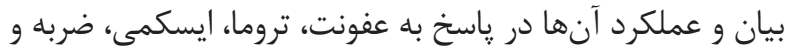

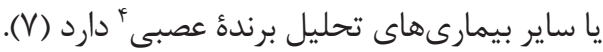

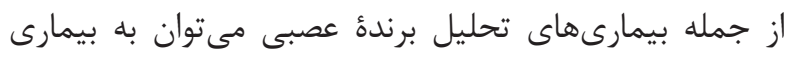

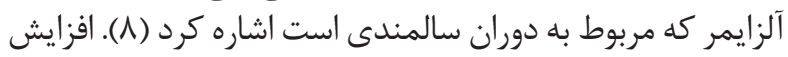

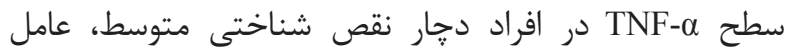

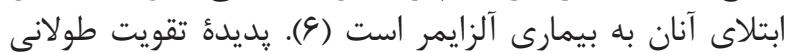

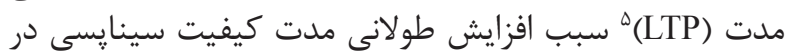

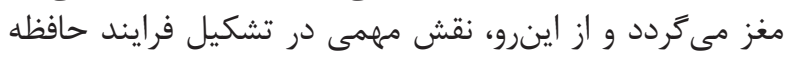

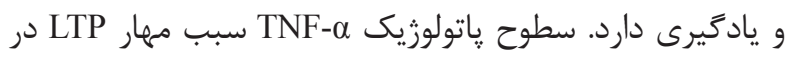

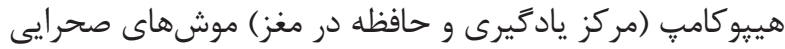

مى مردد (9) (9) (9)

يكى از شناختهترين علل بسيارى از بيمارىها ماند آلزايمر و

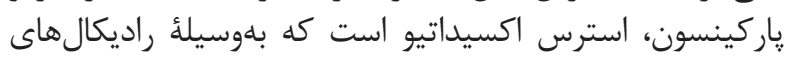

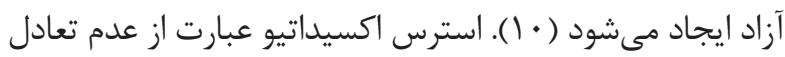

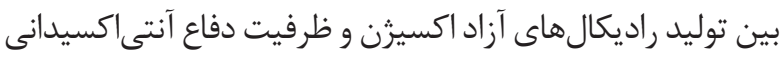

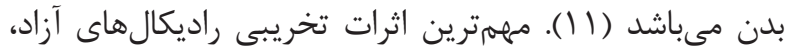

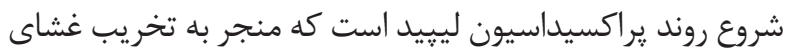

\footnotetext{
${ }^{5}$ Long term potentiation

${ }^{6}$ Blood brain barrier

${ }^{7}$ Sprague Dawley
} 


$$
\text { آناليز آمارى }
$$

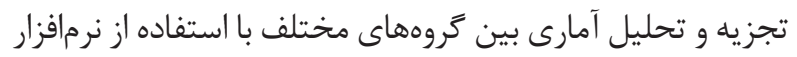

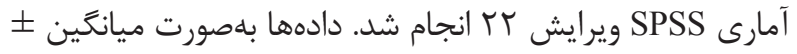

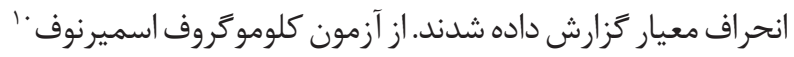

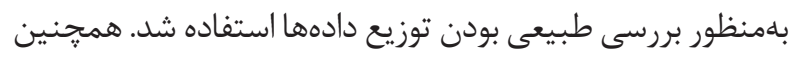

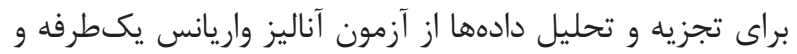

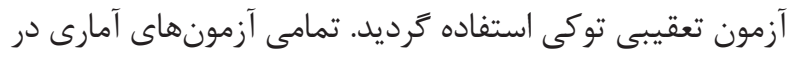

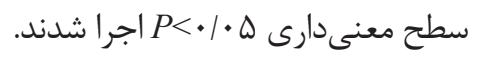

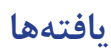

نتايج توصيفى آزمودنىها نشان داد كه بر اثر تمرين، اسيد

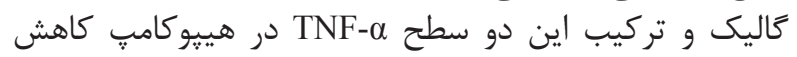

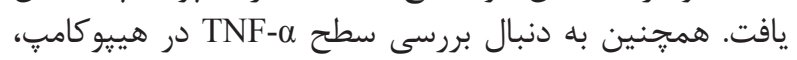

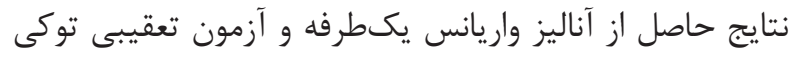

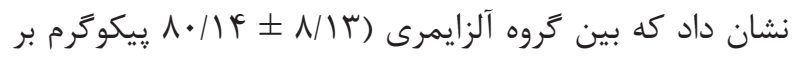

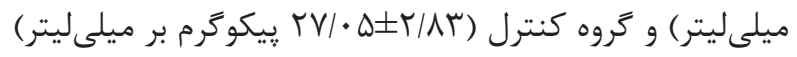

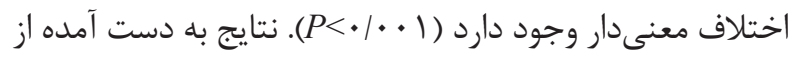

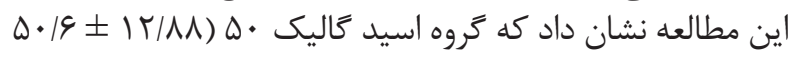

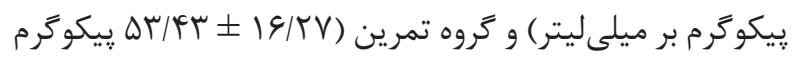

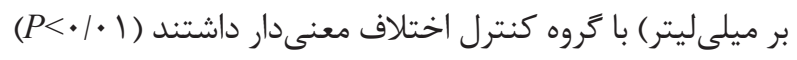

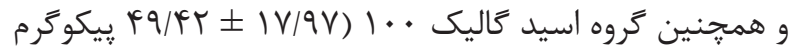

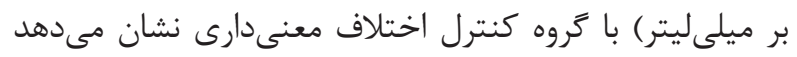

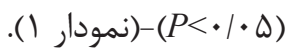

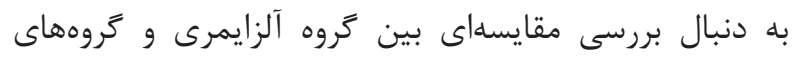

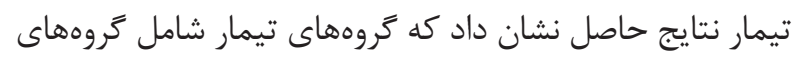

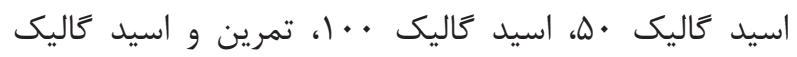

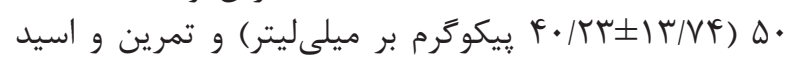

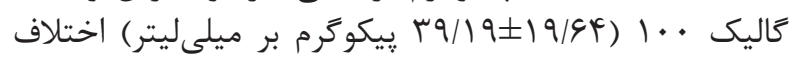

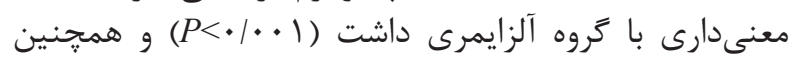

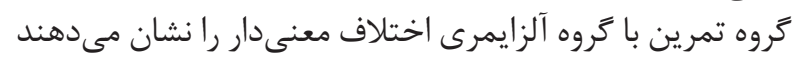

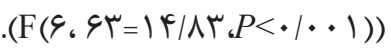

\section{بحث و نتيجه كَيرى}

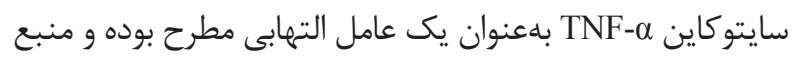

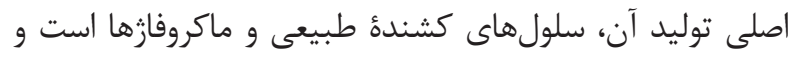

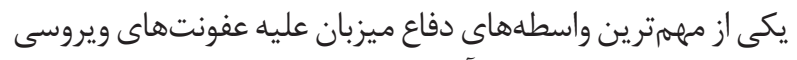

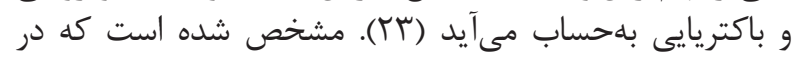

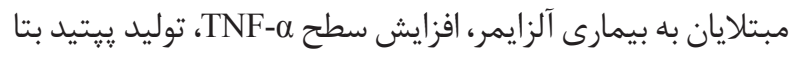

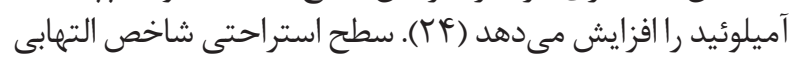

TNF- $\alpha$

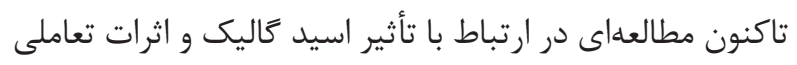

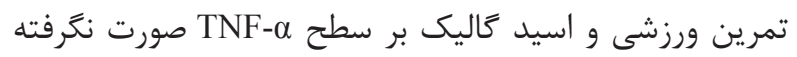

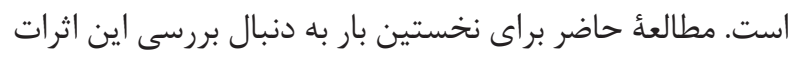
بر سطح TNF-

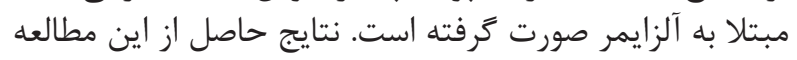

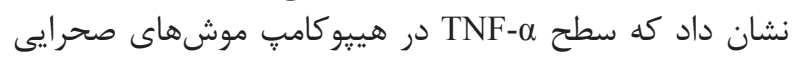

كميتؤ اخلاقى دانشكاه انجام شد.

$$
\text { القاء مدل و تيمار ها }
$$

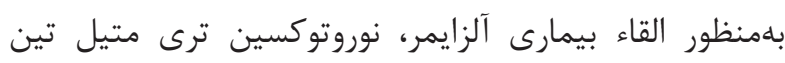

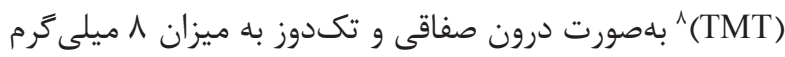

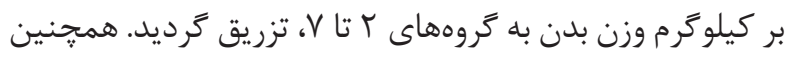

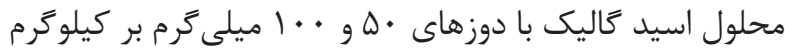

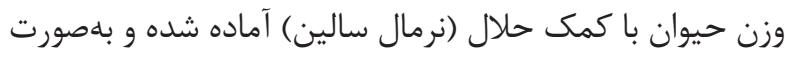

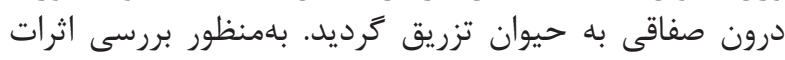

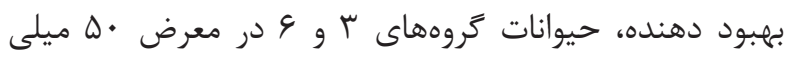

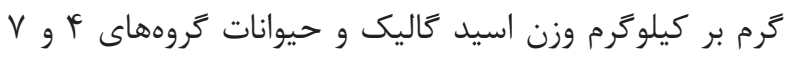

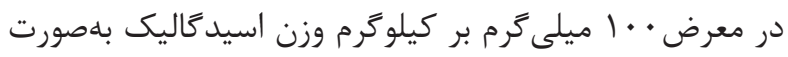
روزانه به مدت دو هفته قرار كرفتيند.

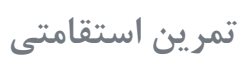

دستورالعمل تمرين استقامتى شامل هشت هفته دويدن فزاينده

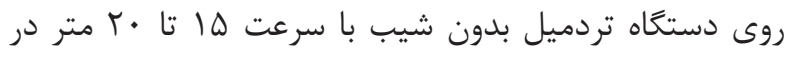

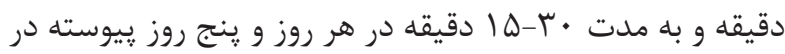

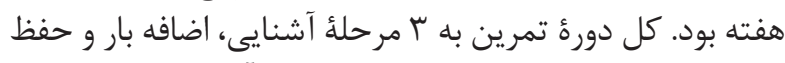

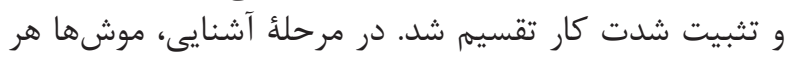

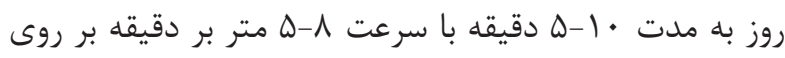

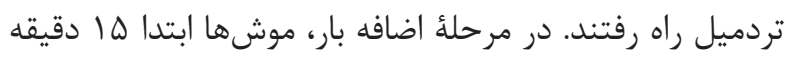

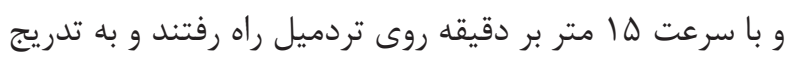

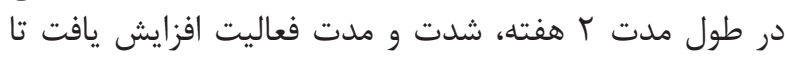

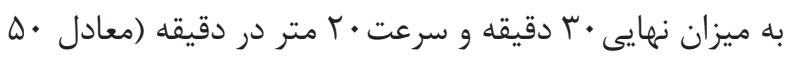

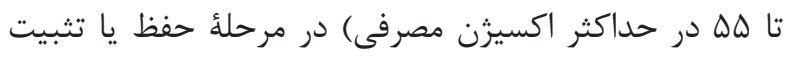

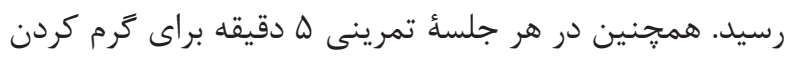

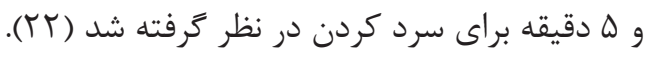

$$
\text { خون كيرى و الايزا }
$$

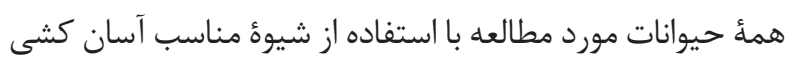

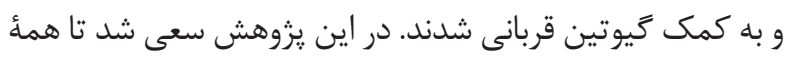

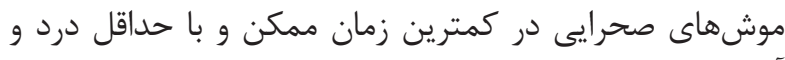

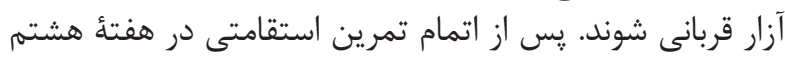

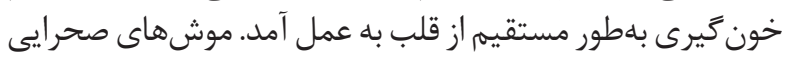

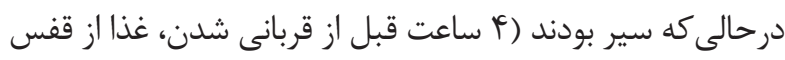

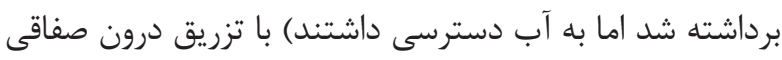

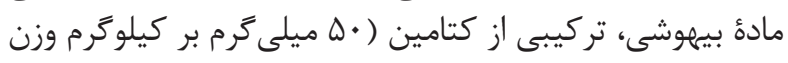

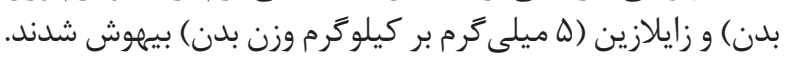

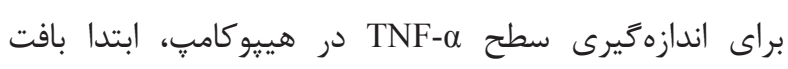

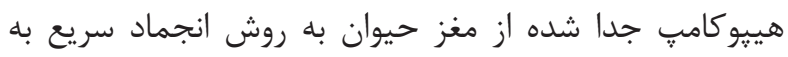

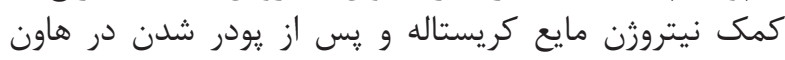

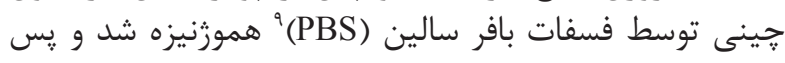

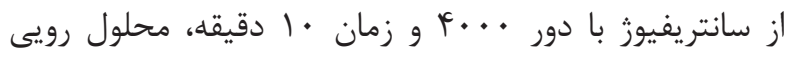

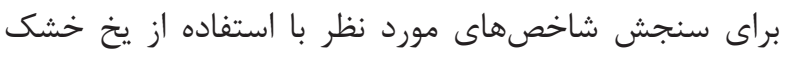

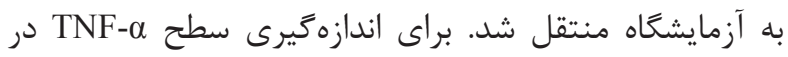
هييوكامٍ در آزمايشكاه از روش الايزا استفاده كرديد.

\footnotetext{
${ }^{8}$ Trimethyltin

${ }^{9}$ Phosphate buffered saline

${ }^{10}$ Kolmogorov-Smirnov test
} 


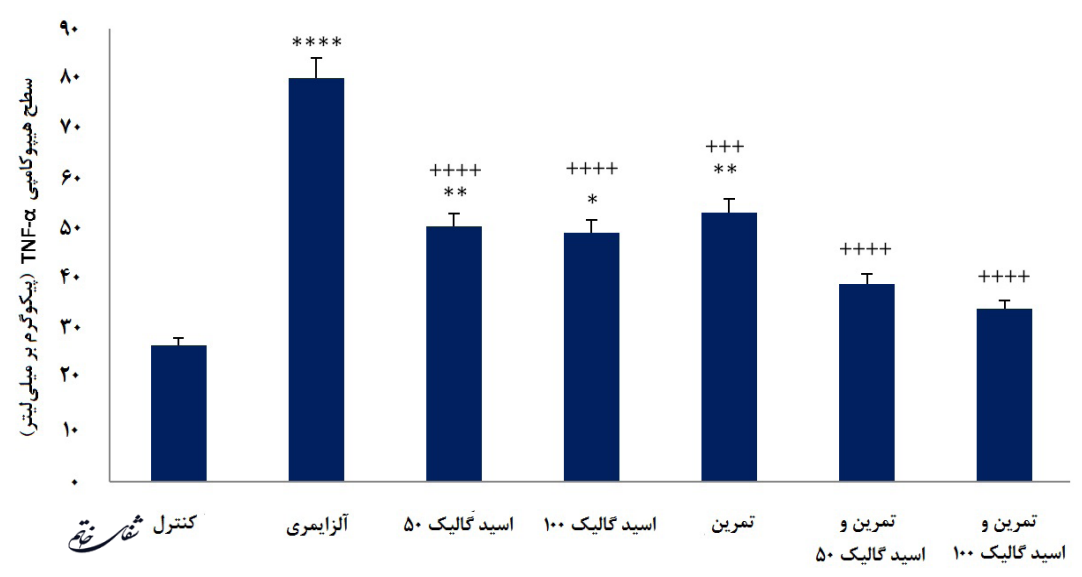

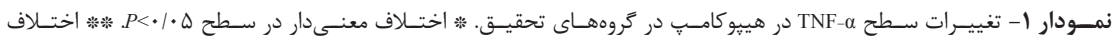

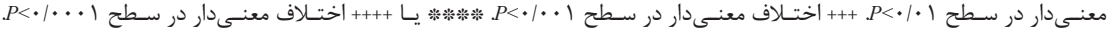

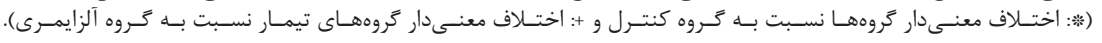

باعث كاهش معنى دار سطوح TNF- دمر مضلات اسكلتى افراد

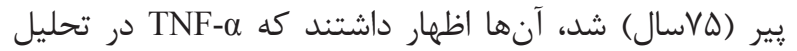

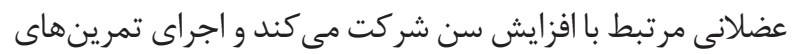

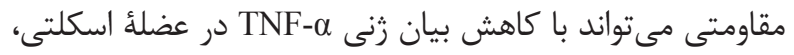
اين فرايند را كاهش دهد (1) (1).

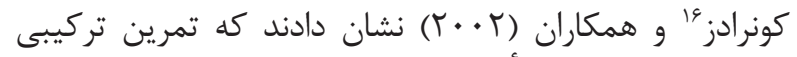

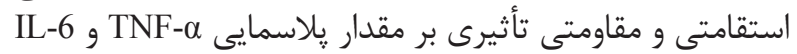

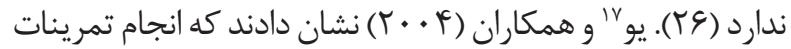

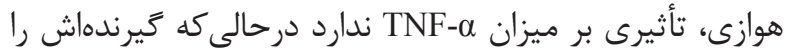

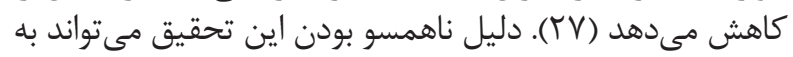

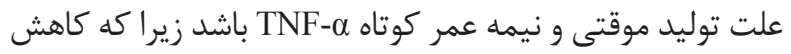

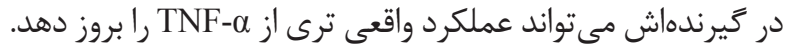

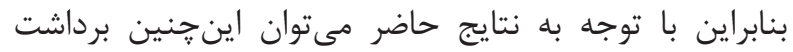

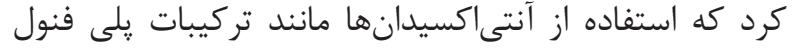

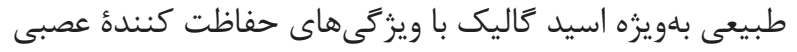

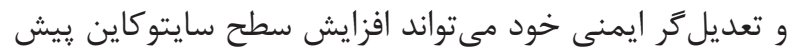

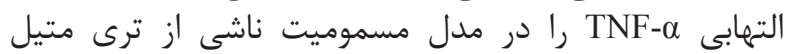

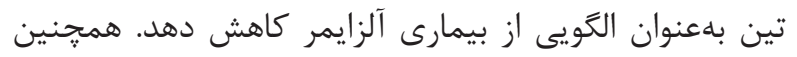

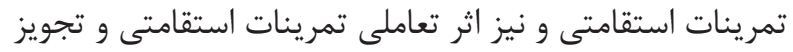

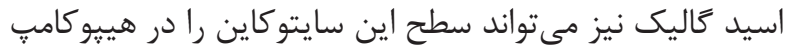
كاهش و سبب تعديل ايمنى در بيمارى آلزايمر كردد.

1. Burns JM, Cronk, BB. Cardiorespiratory fitness and brain atrophy in early Alzheimer's disease. Neurology. 2008; 71(3): 210-6.

2. Brookmeyer R, Johnson E, Ziegler Gk, Arrghi HM. Forecasting the global burden of Alzheimer's disease. Alzheimers Dement. 2007; 3(3): 186-91.

\footnotetext{
${ }^{11}$ Linke

${ }^{12}$ Tsukui

${ }^{13}$ Ryan

${ }^{13}$ Interleukin 6
}

درمان شده با اسيد كاليك در كروههاى اسيد كاليك •ه و

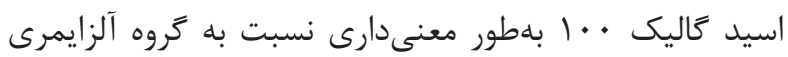

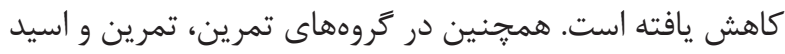

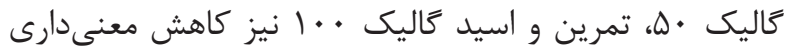

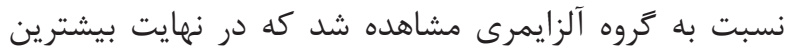

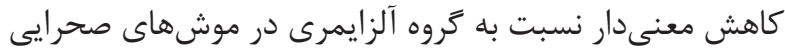

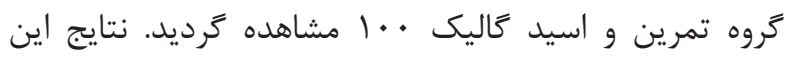

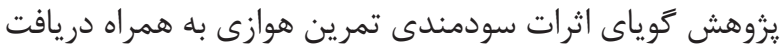

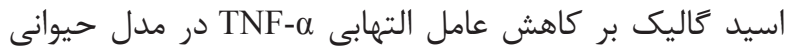

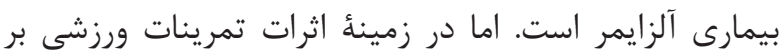
سطح TNF- مطالعاتى انجام كرفته است.

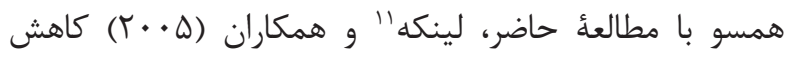
معنى دارى را در ميزان TNF-

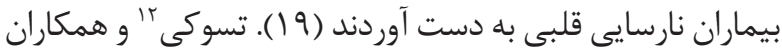

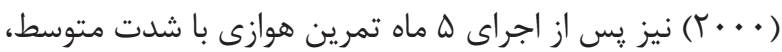

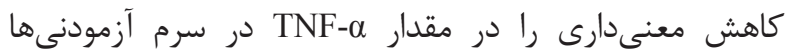

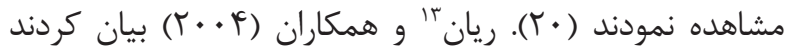

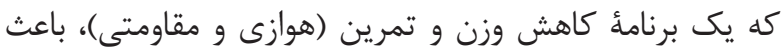

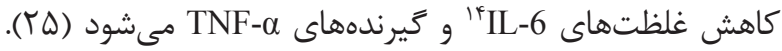

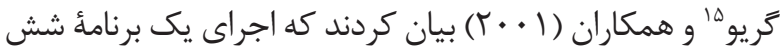
ماهه (سه ماه انعطافيذيرى و سه مان ماه تمرين مقاومتى فزاينده)،

$$
\text { منابع }
$$

3. Solfrizzi V, D'Introno A, Colacicco AM. Circulating biomarkers of cognitive decline and dementia. Clin Chim Acta. 2006; 364: 91-112.

4. Makhija K, Karunakaran S. The role of inflammatory cytokines on the aetiopathogenesis of depression. Aust N Z J Psychiatry. 2013; 47(9): 828-39.

\footnotetext{
${ }^{14}$ Greiwe

${ }^{15}$ Conraads

${ }^{16}$ You
} 
5. Smith LL, Amwar A, Fragen M. Cytokin and cell adhesion molecules associated with high-intensity eccentric exercise . Eur J Appl Physiol. 2000; 82: 61-7.

6. Tarkowski E, Blennow K, Wallin A, Tarkowski A. Intracerebral production of tumor necrosis factor-alpha, alocal neuroprotective agent, in Alzheimer's disease and vascular dementia. J Clin Immunol. 1999; 19(4): 223-30.

7. Pickering M, Cumiskey D, O'Connor JJ. Actions of TNF-alpha on glutamatergic synaptic transmission in the central nervous system. Exp Physiol. 2005; 90: 663-70.

8. Jee YS, Ko IG, Sung YH, Lee JW, Kim YS, Kim SE. Effects of treadmill exercise on memory and c-Fos expression in the hippocampus of the rats' with intracerebroventricular injection of streptozotocin. Neurosci Lett. 2008; 443(3): 188-92.

9. Butler MP, O’Connor JJ, Moynagh PN. Dissection of tumor-necrosis factor-alpha inhibition of long-term potentiation (LTP) reveals a p38 mitogen-activated protein kinase-dependent mechanism which maps to early-but not late-phase LTP. Neuroscience. 2004; 124 : 319-26.

10. Asghari N, Aliakbari M, Dadkhah A. The effects of group logotherapy on decreasing the degree of depression in female olds. MEJDS. 2012; 11(2): 31-8.

11. Mohajeri D, Doostar Y. Antioxidant effect of extract of the grape seed in streptozotocin induced diabetic rats. ZJRMS. 2009; 12(1): 9-14.

12. Koksal E, Gulcin I. Antioxidant activity of cauliflower. Turk J Agric. 2008; 32: 65-78.

13. Mansouri TM, Farbood Y, Sarkaki A. Neuroprotective effects of oral gallic acid against oxidative stress induced by 6 -hydroxydopamine in rats. Food Chem. 2013; 138: 1028-33.

14. Farbood Y, Sarkaki A, Hashemi Sh. The effects of gallic acid on pain and memory following transient global ischemia/ reperfusion in wistar rats. Avicenna J. 2013; 25: 1-12.

15. Fleg JL. Physical activity as anti inflammatory therapy for cardiovascular disease. Prev Cardiol. 2005; 8 (1): 8-10.

16. Gleesen M, Mcdonald WA, Crips AW, Pyne DB, Clancy RL, Fisher PA. The effect on immunity of long -term intensive training in elits swimmers. Clin Exp Immunol. 1995; 102(10): 210-6.

17. Scheett TP, Nemet D, Stoppani J, Maresh CM, Newcomb R, Cooper DM. The effect of endurance-type exercise training on growth mediators and inflammatory cytokines in pre-pubertal and early pubertal males. Pediatr Res. 2002; 52: 491-7.

18. Greiwe JS, Cheng B, Rubin DC, Yarasheski KE, Semenkovich CF. Resistance exercise decreases skeletal muscle tumor necrosis factor $\alpha$ in frail elderly humans. FASEB J. 2001; 15(2): 475-82.

19. Linke A, Adams V, Schulze PC, Erbs S, Gielen S, Fiehn E. Antioxidative effects of exercise training in patients with chronic heart failure: increase in radical scavenger enzyme activity in skeletal muscle. Circulation. 2005; 111: 1763-70.

20. Tsukui S, Kanda T, Nara M, Nishino M, Kondo T, Kobayashi I. Moderate-intensity regular exercise decreases serum tumor necrosis factor-alpha and $\mathrm{HbA1c}$ levels in healthy women. Int J Obes Relat Metab Disord. 2000; 24: 1207-11.

21. Balducci S, Zanuso S, Nicolucci A, Fernando F, Cavallo S, Cardelli P. Anti-inflammatory effect of exercise training in subjects with type 2 diabetes and the metabolic syndrome is dependent on exercise modalities and independent of weight loss. Nutr Metab Cardiovasc Dis. 2010; 20: 608-17.

22. Ooyama K, Wu J, Nosaka N, Aoyama T, Kasai M. Combined intervention of medium-chain triacylglycerol diet and exercise reduces body fat mass and enhance energy expenditure in rats. J Nutr Sci Vitaminol (Tokyo). 2008; 54(2): 136-41

23. Esposito K, Pontillo A, Di Palo C, Giugliano G, Masella M, Marfella R. Effect of weight loss and lifestyle changes on vascular inflammatory markers in obese women: a randomized trial. JAMA. 2003; 289: 1799-804.

24. Blasko I, Marx F, Steiner E, Hartmann T, GrubeckLoebenstein B. TNFalpha plus IFNgamma induce the production of Alzheimer beta amyloid peptides and decreasethesecretionofAPPs.FASEBJ.1999;13(1):63-8.

25. Ryan AS, Nicklas BJ. Reductions in plasma cytokine levels with weight loss improve insulin sensitivity in overweight and obese postmenopausal women. Diabetes Care. 2004; 27: 1699-705.

26. Conraads VM, Beckers P, Bosmans J, De Clerck LS, Stevens WJ, Vrints CJ, et al. Combined endurance/ resistance training reduces plasma TNF- $\alpha$ receptor levels in patients with chronic heart failure and coronary artery disease. Eur Heart J. 2002; 23(23): 1854-60.

27. You T, Berman DM, Ryan AS, Nicklas BJ. Effects of hypocaloric diet and exercise training on inflammation and adipocyte lipolysis in obese postmenopausal woman. J clin Endocrin Metab. 2004; 4: 1739-46. 